\title{
A Multi-objective Evolutionary Method for Dynamic Airspace Re-sectorization using Sectors Clipping and Similarities
}

\author{
Jiangjun Tang*, Sameer Alam ${ }^{\dagger}$, Chris Lokan ${ }^{\ddagger}$, and Hussein A. Abbass ${ }^{\S}$ \\ * School of Engineering and Information Technology \\ UNSW@ADFA, Canberra, Australia \\ Email: j.tang@adfa.edu.au \\ †Email: s.alam@adfa.edu.au \\ †Email: c.lokan@adfa.edu.au \\ Email: h.abbass@adfa.edu.au
}

\begin{abstract}
Dynamic Airspace Sectorization (DAS) is a future concept in Air Traffic Management. Its main goal is to increase airspace capacity by reshaping - thus optimizing - airspace sector boundaries based on the specifics of different air traffic situations, weather conditions and other factors. The primary objective for the optimization is to balance and reduce the workload of Air Traffic Controllers (ATCs). Many researchers have made efforts in this topic in the past years. However, air traffic changes continually, and DAS has to be adaptive to each change; be it in terms of aircraft density, dynamic routes, fleet mix, etc. Therefore, instead of sectorizing the airspace each time a change occurs, we should re-sectorize it by maintaining maximum similarities between each sectorization.

In this paper, we propose a multi-objective evolutionary computation methodology to re-sectorize an airspace. We use a similarity measure between the existing sectorization and the resectorization as an objective to maximize during the evolution. We test the methodology with different air traffic conditions with four objective functions: minimize ATC task load standard deviation, maximize average flight sector time, maximize the minimum distance between traffic crossing points and sector boundaries, and maximize the similarity of two airspace sectorizations. Experimental results show that our re-sectorization method is able to perform airspace re-sectorization under different changes in the air traffic, while satisfying the predefined objectives.
\end{abstract}

\section{INTRODUCTION}

Large organizations such as Eurocontrol in Europe, FAA in the USA, and Airservices in Australia are working towards improving the future airspace. Dynamic Airspace Sectorization (DAS) is one of the future Air Traffic Management (ATM) Concepts that is being proposed to manage the increase in airspace capacity while managing the workload on Air Traffic Controllers (ATCs). The primary focus of DAS is ATCs' workload. One primary question is how to maintain a balance in an ATC workload through the sectorization process. It is then obvious that a dramatic change from one sectorization to another is not recommended. It overloads the ATC by exposing an ATC to very unfamiliar sector shapes. Therefore, there is an urge for Dynamic Airspace Re-sectorization techniques.

In the last decade, a large number of researchers have been working on 2D airspace sectorization. A common approach is to cluster the flights based on their trajectories [1]. Air traffic flow is composed of 4D aircraft trajectories; each trajectory is a temporal-spatial data set. Flight trajectory clustering groups flight track positions together to sectorize the airspace. Sector boundaries are then formed around the groupings of flight route segments.

Some authors formulated the problem as a Mixed Integer Programming (MIP) model. They discretized the airspace into hexagonal cells. These cells are then clustered based on the estimated controller task load in each cell ${ }^{1}$ and a connectivity measure [2], [3].

Another approach is to use Voronoi Diagrams. Here, the airspace is partitioned and a meta heuristic technique, such as genetic algorithms, is used to optimize the partitions [4], [5], [6]. The airspace is decomposed using Voronoi Diagrams into subdivisions around given generating points. All air traffic footprints (such as radar points) in a specific region of the airspace, and which are associated with a specific generating point are closer to that generating point than any other generating point. The task of a genetic algorithm is to discover the location of these generating points to optimize the problem.

Some DAS methods were also introduced for a three dimensional airspace. To partition an airspace, an AgentBased Model (ABM) [7] is combined with a genetic algorithm (GA). GA is used to determine the initial locations of agents, and the agent-based model is used to cluster the cells. The airspace is tiled with uniform grid cells in three dimensions. A number of drawbacks can be identified with this approach [8]; these include violation of the convexity constraint associated with the shape of sectors, and the right prism constraint which guarantees that a $2 \mathrm{D}$ projection of the $3 \mathrm{D}$ airspace on a controller screen does not confuse the controller. In

\footnotetext{
${ }^{1}$ The terminology "workload" was used in most DAS approaches. However, "workload" is not measured by cognitive metrics or other factors directly from ATC but by the air traffic data, such as aircraft counts and traffic loads. Although they reflect the ATC workload somehow, they are really measurements of "task load" rather than "workload". Therefore, "task load" is used instead of "workload" in this paper.
} 
addition, the $\mathrm{ABM}$ has a high computational cost. In our previous work [8], we developed four new models for 3D DAS to overcome all limitations that exist in the ABM, and we also found that two of these models have more balanced performance and efficiency.

However, all mentioned methodologies focused on dynamic airspace sectorization rather than continuous airspace resectorization. Methodologies for re-sectorizing an airspace according to the air traffic situations are lacking in the literature. The main difficulty of re-sectorization is to minimize the changes to the sector boundaries between sectorizations, because training an ATC to be familiar with all sector characteristics, including geographic characteristics, airways, navigation waypoints, traffic flows, and procedural rules, is a time consuming process. Smaller changes to sector boundaries make it easier for ATC to manage the air traffic during dynamic airspace re-sectorization.

In this paper, we present an approach based on evolutionary computation for Dynamic Airspace Re-sectorization in a 2D airspace. The experimental results show that our approach can satisfy the predefined objectives and sector design constraints, and it also can maintain similarities of sector boundaries between consecutive sectorizations.

\section{Methodologies for Dynamic Airspace RE-SECTORIZATION}

In this section, we present the methodologies for Dynamic Airspace Re-sectorization in 2D airspace, focusing mainly on the method of measuring similarity of airspace sectorizations and evolutionary optimization technology. First, the definitions used in Dynamic Airspace Sectorization and Re-sectorization are described in the next section.

\section{A. Problem definitions}

For Dynamic Airspace Sectorization and Re-sectorization, the inputs are a given airspace and a set of flight trajectories, and the output is a set of partitioned sectors that satisfy specified objectives, such as balancing ATC workload.

In this paper, our proposed model works on a continuous airspace. The continuous airspace is defined as $R(X)$, where $X=\left\{x_{1}, x_{2}, x_{3}, \ldots x_{n}\right\}$ is all points included in $R . x_{j}=$ $\left(l a t_{j}, \operatorname{lon}_{j}, a l t_{j}\right)$ is a 3 -tuple recording latitude, longitude, and altitude.

The air traffic is a set of trajectories: $T=\left\{t_{i}\right\}_{i=1}^{N}$, where

$$
t_{i}=\left(x_{i j}, \text { spd }_{i j}, \text { time }_{i j}\right)_{j=k l 1}^{M}
$$

$t_{i}$ is the trajectory of flight $i$, consisting of a set of ordered points $x_{i j}=\left\{l a t_{i j}, l_{o n} n_{i j}, a l t_{i j}\right\}$ and the speed $\left(s p d_{i j}\right)$ and time $\left(\right.$ time $\left._{i j}\right)$ associated with each point.

In airspace sectorization, only the trajectories located within $R$ are considered. Therefore,

$$
\forall i \text { and } \forall j: x_{i j} \in R
$$

The airspace $R$ is partitioned into $K$ sectors $S_{k},(k=$ $1,2,3, \ldots, K) . S(\hat{X})_{k}$ is used as a sector notation for the continuous approach, where $S$ is the superset of $\hat{X}$ and $\hat{X}$ is a subset of $X$. Additionally, for sector definitions,

$$
R=\bigcup_{k=1}^{K} S_{k}
$$

and

$$
S_{i} \cap S_{j}=\emptyset ; i \neq j ; i, j \in\{1,2,3, \ldots K\}
$$

After the space is partitioned as $S_{k}$, all points $x_{i j}$ in $T$ must satisfy

$$
\forall i \text { and } \forall j: \text { IF } x_{i j} \in S_{k} \text { THEN } x_{i j} \notin\left(R \backslash S_{k}\right)
$$

For Dynamic Airspace Sectorization and Re-sectorization, it is necessary to model the ATC task load mathematically. In this paper, the task load of a sector is measured by the count of traffic hits within the sector during a given period. Equation 1 describes the task load measurement we use:

$$
W_{k}=\sum_{i=1, x_{i j} \in S_{k}}^{N}\left|t_{i}\left(x_{i j}\right)\right|
$$

$W_{k}$ is the task load of a sector $S_{k}$, which is measured by the count of $x_{i j}$ within $S_{k}$ from all trajectories $t_{i}$.

Sector flight time is also measured in this paper. It contributes to the alignment between sectors and traffic flows, which minimizes the traffic flow cut and therefore reduces the handover task load for ATCs. It is modelled as Equation 2:

$$
S F T_{k}=\frac{\sum_{i=1}^{N} \sum_{j=1, x_{i j} \in S_{k}, x_{i j+1} \in S_{k}}^{M}\left(\text { time }_{i j+1}-t_{i m e_{i j}}\right)}{\left|T\left(t_{i}\right)\right|_{x_{i j} \in S_{k}}}
$$

The sector flight time is derived from the time stamps $\left(\right.$ time $\left._{i j}\right)$ of its continuous radar signatures in $t_{i}$, which contain positions $\left(x_{i j}\right)$ at time $_{i j}$ located within the sector $\left(S_{k}\right) . T\left(t_{i}\right)$ is a set of trajectories in the space $R$ as described above. Therefore, the count of all trajectories $t_{i}$ that have some parts $x_{i j}$ located within a sector $S_{k}$ is the number of flights flying within $S_{k}$. According to these, the sector flight time for a sector can be produced by Equation 2 .

The minimum distances $(D)$ between traffic crossing points and sector boundaries is considered in this paper as well. A larger distance between crossing points and sector boundaries gives an ATC more time to respond in order to resolve a potential conflict. The crossing points of air traffic flows $(C P)$ are identified based on the 4D flight trajectories $\left(t_{i}\left(x_{i j}\right)\right)$, where the two flight footprints satisfy Equation 3:

$$
\begin{aligned}
& \operatorname{Dist}\left(x_{p u}\left(\text { lat }_{p u}, \text { lon }_{p u}\right), x_{q v}\left(\text { lat }_{q v}, \text { lon }_{q v}\right)\right) \leq 5 n m \bigvee \\
& \mid x_{p u}\left(\text { alt }_{p u}\right)-x_{q v}\left(\text { alt }_{q v}\right) \mid \leq 1000 \mathrm{ft} \bigvee \\
& \mid \text { time }_{p u}-\text { time }_{q v} \mid \leq 300 \mathrm{sec}
\end{aligned}
$$

$x_{p u}$ and $x_{q v}$ are the locations of two flights $p$ and $q$ at time $e_{p u}$ and time $_{q v}$. As shown in Equation 3, we take 5nm lateral distance and $1000 \mathrm{ft}$ vertical separation within 300 seconds time window as a threshold to identify the crossing points between flights. The two flight footprints are treated as the crossing points of traffic flows. Then the minimum distance 
between a traffic crossing point and the corresponding sector boundaries for a sector can be calculated as:

$$
D_{k}=\min _{i=1}^{P}\left(\operatorname{Dist}\left(C P_{i}, \text { Boundaries }\left(S_{k}\right)\right)\right) I F C P_{i} \in S_{k}
$$

where $P$ is the total number of crossing points identified in the given airspace $R$ and the minimum distance $\left(D_{k}\right)$ is calculated for the sector $S_{k}$ only when $C P_{i}$ is inside it.

\section{B. Airspace Sectorization Method}

A Constrained Voronoi Diagram-based model (CVDM) for 3D Airspace Sectorization has been developed in our previous work [8]. A Voronoi diagram is a special kind of decomposition of a metric space, determined by distances to a specified discrete set of objects (sites) in the space. A Voronoi diagram guarantees convexity for each Voronoi cell. Although Voronoi Diagrams can partition 3D space, they can't guarantee to satisfy right prism constraint. In this model, Voronoi Diagram is only used for lateral partitioning; vertical division is achieved by the vertical value of Voronoi sites, to satisfy the right prism constraint. Therefore, it is called Constrained Voronoi Diagram based Model.

In this paper, we use the Constrained Voronoi Diagram Model (CVDM) in 2D, in which the third dimension (vertical) is switched off. Therefore, we only consider the lateral points as the Voronoi sites to partition airspace and to satisfy the airspace sectorization objectives.

\section{Similarity of Sector Shapes}

In our approach, the similarity of Sector shapes is measured by the overlapping of two sector shapes from two airspace sectorizations. One current sector may overlap with multiple previous sectors and multiple current sectors may also overlap with one previous sector, as illustrated in Figure 1. The boxes with dashed boundaries are the sectors from the previous airspace sectorization and the boxes with solid boundaries are the sectors from the current results.

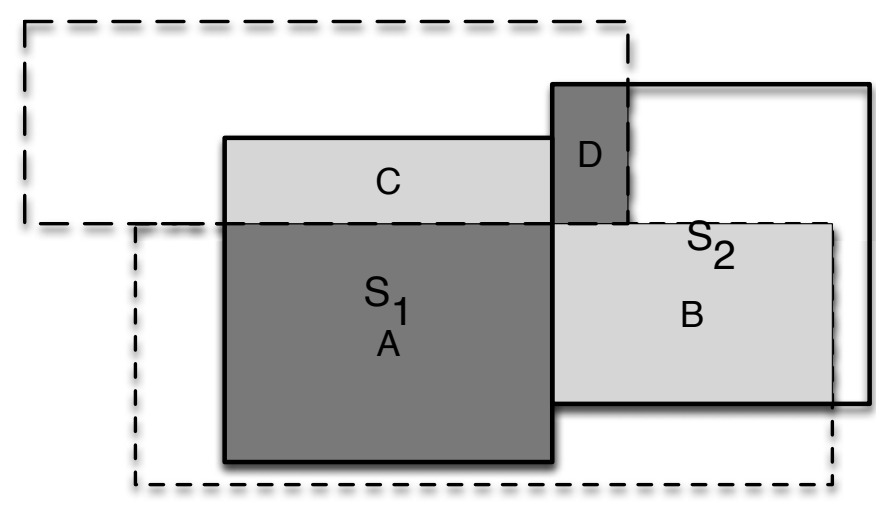

Fig. 1: An Example of Overlapping between Sectors from Resectorization and Original Sectors

In the example, sector $S_{1}$ has overlapping areas ( $A$ and $C$ ) with two previous sectors; $S_{2}$ has overlapping areas ( $B$ and
$D)$ with those previous sectors. Only the maximum area $(A)$ is considered as the overlapping area of $S_{1}$ with the previous sectors. The figure shows that both $A$ of $S_{1}$ and $B$ of $S_{2}$ belonged to the same previous sector but $A$ is bigger than $B$. Therefore, although the overlap $(B)$ is bigger than the area $(D)$ for sector $S_{2}, D$ is considered as the overlapping area of $S_{2}$ with the previous sectors.

The overlapping between two sector shapes is generated by Weiler-Atherton Clipping algorithm [9] and then the area is calculated. The maximum overlapping area of a sector with all sectors in the previous airspace sectorization is used as the similarity of this sector to the previous sectorization, which is calculated based on Equation 5:

$$
a_{k}=\max _{j=1}^{K}\left(S_{k, d+1} \cap S_{j, d}\right) \quad k \in[1, K]
$$

where $S_{j, d}$ is one of the sectors generated from the previous airspace sectorization $(d)$ and $S_{k, d+1}$ is a sector produced in the current airspace sectorization $(d+1) . a_{k}$ is the maximum overlapping area of $S_{k, d+1}$ with all sectors from the previous airspace sectorization.

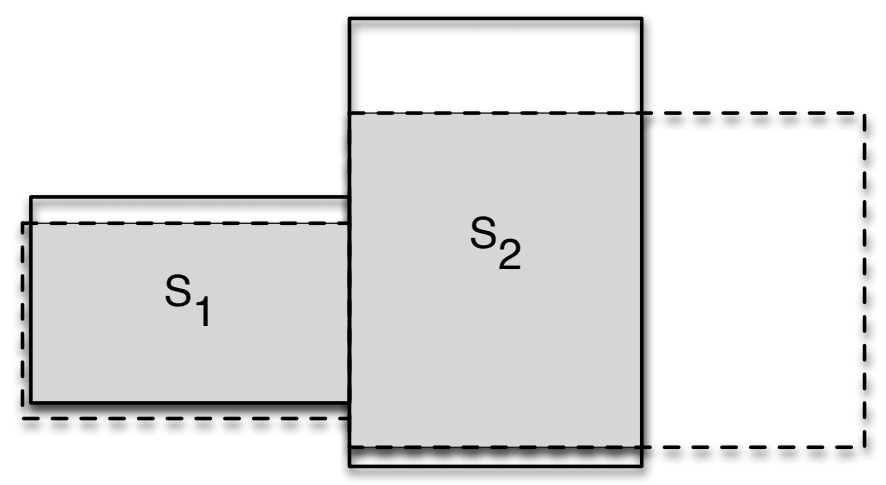

Fig. 2: An Example of Similarity Measurement by the Ratio of Overlapping Area and Origin Sector

To find out if two airspace sectorizations are similar or not, the absolute value of maximum overlapping area is not accurate enough as the similarity metric, because the areas of each sector are usually different. For example, in Figure 2, $S_{1}$ is more similar to the original sector than $S_{2}$ although the overlapping area of $S_{2}$ with the original sector is bigger than $S_{1}$. Therefore, we use a normalized value to represent the similarity of a sector to the original sector, as calculated by Equation 6:

$$
r_{k}=\frac{a_{k}}{S_{j, d}}
$$

where $S_{j, d}$ is the original sector which has the biggest overlap with the current sector $S_{k}$ and $a_{k}$ is the area calculated by Equation 5. Therefore, $r_{k}$ is the ratio between the overlap area and the original sector area. In our approach, the measurement of the similarity of two individual sectors is based on $r_{k}$. A higher value of $r_{k}$ means the current sector is more similar to the original sector. 


\section{DAS as a Multi-ObJective Optimization PROBLEM}

In Dynamic Airspace Sectorization, we have four objectives:

- Minimizing task load standard deviation, aiming to minimize the task load variance between sectors with a given sector number.

- Maximizing average sector flight time, aiming to maximize the time for which flights stay within a sector and to align the traffic flow with sectors in order to reduce the flow cuts.

- Maximizing distance between traffic flow crossing points and sector boundaries, aiming to maximize the minimum distance between the flow crossing points and sector boundaries to ensure enough time for an ATC to respond to resolve a potential conflict.

- Maximizing the similarity of two airspace sectorizations, aiming to minimize the sector shape changes between two airspace sectorizations and thus make it easier for ATC to manage the air traffic within similar sector configurations.

The first two objectives do not conflict directly, but there is implicit conflict between them. Equation 11 shows that to maximize the flight time within a sector, the sector should have more traffic hits from the same flights. That means a sector can achieve longer sector flight time by aligning its shape with the major traffic flows, to minimize the flow cut. This may cause sectors to encapsulate as much traffic flow as possible, but this will violate the objective of balancing ATC task load.

Therefore it is a multi-objective problem, and there is no straightforward way apart from multi-objective optimization to generate a trade-off set for the given objectives.

\section{A. Multi-objective Optimization by NSGA-II}

NSGA-II is an efficient multi-objective evolutionary algorithm based on the Pareto optimal concept. It applies nondominated sorting to rank individuals, and uses crowding distance to maintain diversity without specifying any additional parameters. The improved sorting approach reduces the computational complexity. It also uses an elitist strategy to expand the sample space. NSGA-II is widely used in many applications to solve multi-objective optimization problems.

NSGA-II is adopted as the optimization algorithm here, as follows:

1) Randomly initialize the first population

2) Continue the following steps until the termination condition (maximum generation reached) is satisfied:

a) Airspace sectorization by proposed models

b) Evaluate the fitness functions for each individual

c) Sort the population and produce offspring by crossover and mutation

3) Represent the optimal solutions

\section{B. Chromosome Representation and Operators}

The inputs to our airspace sectorization approaches are the same: a list of points specifying latitude and longitude in an airspace. Each chromosome contains a list of 2D points. Each point has values of latitude and longitude as shown in Figure 3. These points represent Voronoi sites for the 2D CVDM.

\begin{tabular}{|l|l|l|l|l|l|l|l|l|l|}
\hline Lat $_{1}$ & Lon $_{1}$ & Lat $_{2}$ & Lon $_{2}$ & Lat $_{3}$ & Lon $_{3}$ & $\ldots$ & $\ldots$ & Lat $_{n}$ & Lon $_{n}$ \\
\hline
\end{tabular}

Fig. 3: Chromosome Representation in NSGA-II for 3D Airspace Sectorization

For recombination, we use the standard the simulated binary crossover (SBX) operator [10] with cross-over probability $p_{c}=0.9$ and a distribution index of $\eta_{c}=15$.

Equations 7 and 8 detail the mutation operator employed by the genetic algorithm (see [11]):

$$
\begin{gathered}
y_{i}=x_{i}+\left(x_{i}^{U}-x_{i}^{L}\right) \delta_{i} \\
\delta_{i}= \begin{cases}\left(2 r_{i}\right)^{1 /\left(\eta_{m}+1\right)}-1 & \text { if } r_{i}<0.5 \\
1-\left|2 r_{i}\right|^{1 /\left(\eta_{m}+1\right)} & \text { otherwise }\end{cases}
\end{gathered}
$$

where $x_{i}$ is the value of the $i^{\text {th }}$ parameter selected for mutation; $y_{i}$ is the result of the mutation; $x_{i}^{L}$ and $x_{i}^{U}$ are the lower bound and the upper bound of $x_{i}$ respectively, and $r_{i}$ is a random number in $[0,1] ; \eta_{m}$ is a control parameter ( $\eta_{m}=20$ in our study).

\section{Fitness Function}

With four objectives, we specify four fitness functions to be used by NSGA-II.

The first objective is modelled as the standard deviation of the sectors' task load, as shown in Equation 9:

$$
F(W)=\sqrt{\frac{1}{K} \sum_{k=1}^{K}\left(W_{k}-W_{\text {avg }}\right)^{2}}
$$

$W_{k}$ is the task load of sector $S_{k}$, which is derived from Equation 1. $W_{\text {avg }}$ is the average task load of all sectors in the given airspace. $K$ is the number of desired sectors, which is predefined as an input to the optimization. $W_{\text {avg }}$ is calculated by Equation 10:

$$
W_{a v g}=\frac{\sum_{i=1, x_{i j} \in R}^{N}\left|t_{i}\left(x_{i j}\right)\right|}{K}
$$

The second objective is modelled as the average sector flight time of all sectors, as shown in Equation 11. $S F T_{k}$ is the sector flight time of sector $S_{k}$, produced by Equation 2:

$$
F(S F T)=\frac{\sum_{k=1}^{K} S F T_{k}}{K}
$$

The third objective is modelled as the minimum distance between traffic flow crossing points and sector boundaries among all sectors, as shown in Equation 12:

$$
F(D)=\min _{i=0}^{K} D_{i}
$$

where $D_{i}$ is calculated by Equation 4. $F(D)$ is the minimum distance among all sectors which excludes the distances between the crossing points and the airspace boundaries. 
These three objectives are used in our DAS model first.

After the optimized sectorization results are generated, the fourth objective (to minimize the sector boundary changes during the re-sectorization) is added into our Dynamic Airspace Re-sectorization Model. As mentioned in Section II-C, the similarity of individual sectors is measured by the ratio $\left(r_{k}\right)$ between the overlapping area and original sector area. To maintain the similarity of all sectors in two airspace sectorizations is to improve the minimum $r_{k}$ from all sectors. Therefore the fourth objective is modeled as Equation 13:

$$
F(r)=\min _{k=1}^{K}\left(r_{k}\right)
$$

\section{EXPERIMENTS AND RESULTS}

\section{A. Experiments Design}

The framework of our experiments is presented in Figure 4.

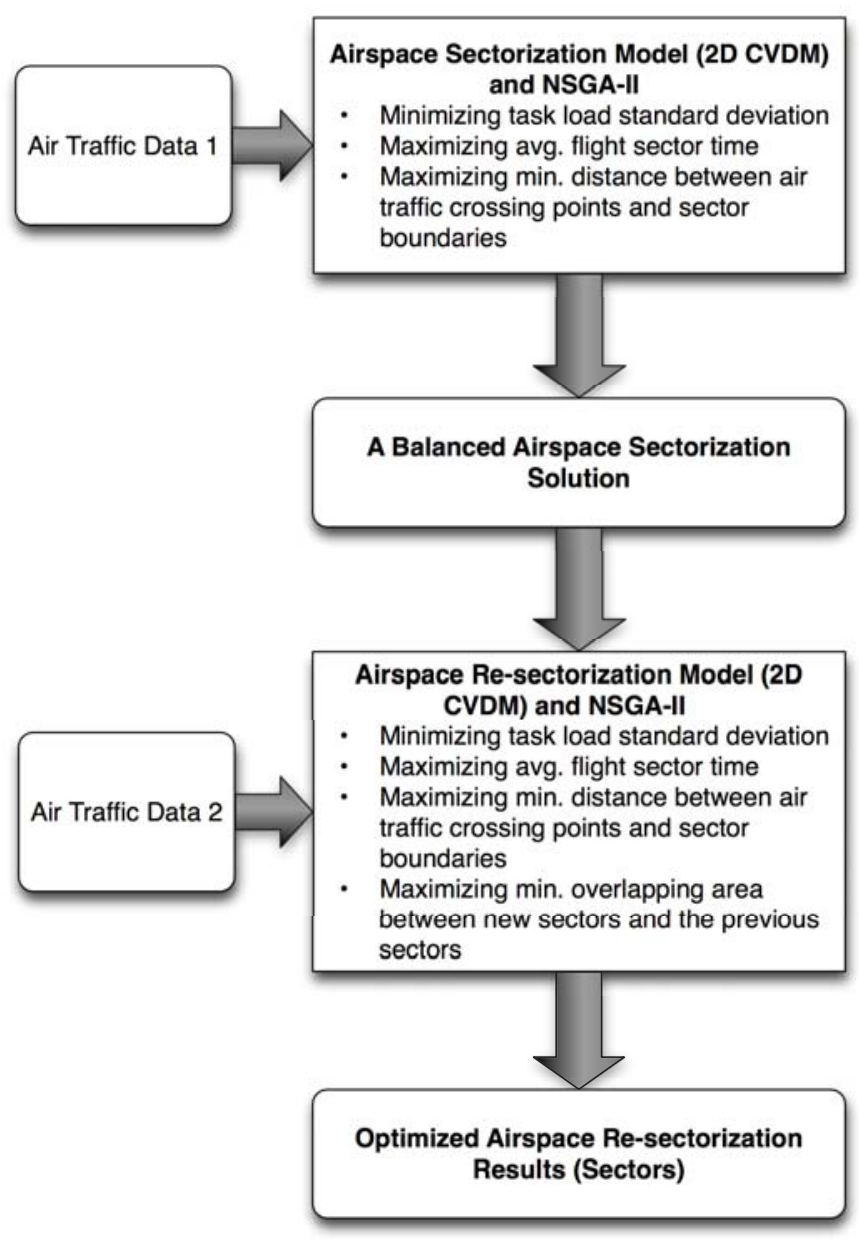

Fig. 4: The Framework of Dynamic Airspace Re-sectorization Experiment

There are two components in our experiments. The first is to perform an airspace sectorization by our 2D CVDM, with the following three objectives:

- Minimizing task load standard deviation.
- Maximizing average sector flight time.

- Maximizing distance between traffic flow crossing points and sector boundaries.

From the Pareto front of the first component, a balanced solution is picked. This solution, as the original sector configuration, is the input to the second component. Then the second component is to undertake an airspace re-sectorization by 2D CVDM, aiming and to satisfy not only the above three objectives but also to maximize the similarity of the current output with the original sectors.

Therefore, each component needs different air traffic data to conduct the experiments. The air traffic data and experiments settings are explained in the following sections.

1) Air Traffic Data: Two sets of air traffic are simulated by ATOMS [12] at the same airspace location for our airspace re-sectorization experiments. The simulated air traffic hits are visualized as color maps in Figure 5. The first set of air traffic is used in our 2D CVDM for airspace sectorization, and the second set is used in our 2D CVDM for airspace resectorization.

Although the total traffic hits of both air traffic data sets are similar (Air Traffic 1 has 23619 traffic hits and Air Traffic 2 has 23308 traffic hits), the traffic patterns are quite different. The first set has very clear air traffic flows and is congested along these flows, as illustrated in Figure 5a, while the second set is more distributed in the airspace with some congestion at the top-right and bottom-left corners, as shown in Figure 5b. These characteristics of the air traffic increases the difficulty to maintain the sectorization similarity during airspace resectorizations and help us to investigate our approach.

2) NSGA-II Settings: We expect to partition the given airspace into 10 sectors according to the air traffic, therefore, there are 10 points (20 real numbers) in one chromosome. The population sizes for both airspace sectorization and airspace re-sectorization are 500 in our experiments. The number of generations for both is 500. Other settings including crossover and mutation are set as described in Section III-A. Both components are run with 10 seeds.

3) A Balanced Airspace Sectorization Solution: From the Pareto set resulting from the first component, we first selected all task load balanced solutions which satisfy these conditions:

$$
\begin{aligned}
& F(W)<=W_{e} \\
& W_{e}=W_{a v g} \times \varepsilon_{w}
\end{aligned}
$$

where $\varepsilon_{w}$ is set as 0.2 (as in our previous work [8]). Therefore, all solutions with the task load standard deviation less than 472.38 are selected because the average task load of Air Traffic 1 is 2361.9 for 10 sectors.

A balanced solution is one that has the highest weighted sum of all normalized objective values. The objectives are weighted equally. For Air Traffic 1, the balanced solution $(F(W)=280.41$ traffic hits, $F(S F T)=620.82 \mathrm{sec}$, and $F(D)=0.89 \mathrm{~nm}$ ) is selected from these acceptable solutions; it is visualized in Figure 6. The sector shapes are taken as the input to the second part for similarity measurements. When the same sectors are used for Air Traffic 2, the four objectives 


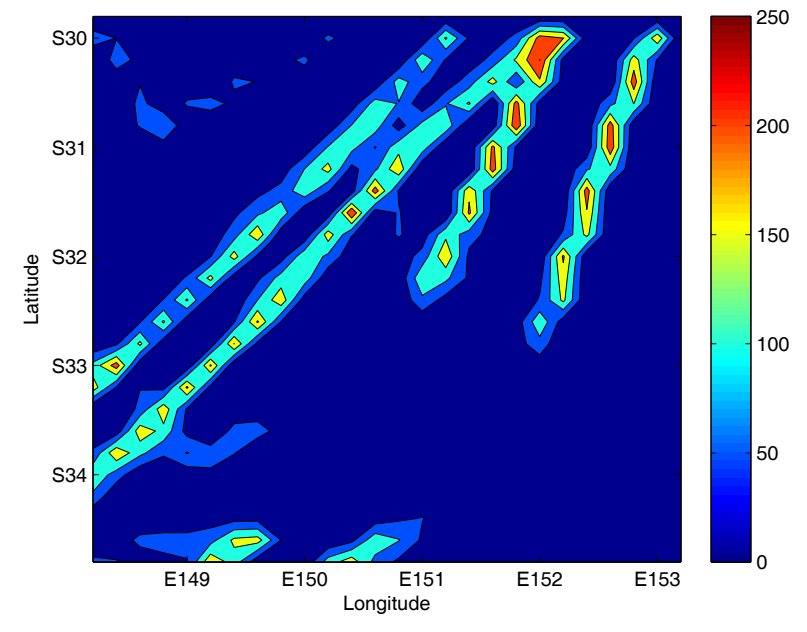

(a) Air Traffic 1(Total Traffic Hits: 23619)

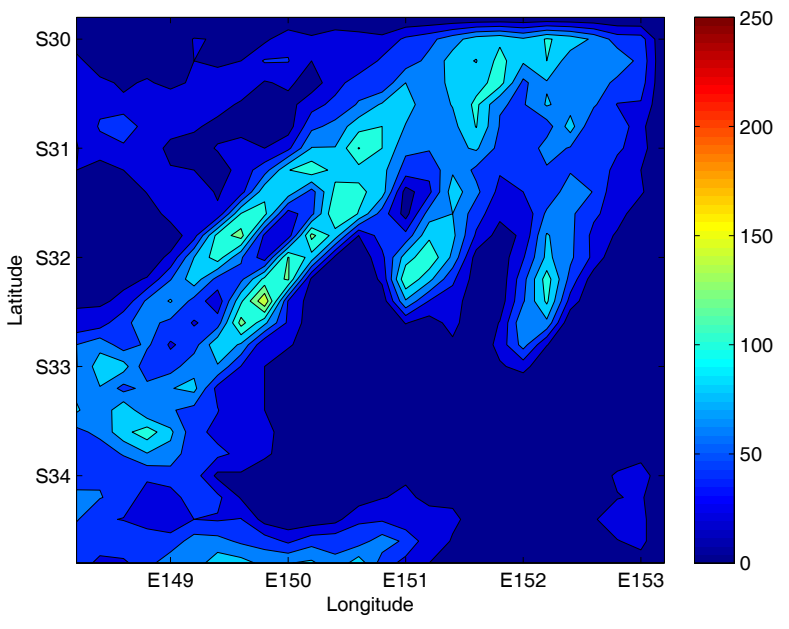

(b) Air Traffic 2 (Total Traffic Hits: 23308)

Fig. 5: The Air Traffic Used in CVDM for Dynamic Airspace Sectorization and Re-sectorization. The Traffic flow on the left hand (a) has clear air but congested traffic flows, whereas the Traffic flow on the right hand (b) is more distributed with some congestion at the top-right and bottom-left corners.

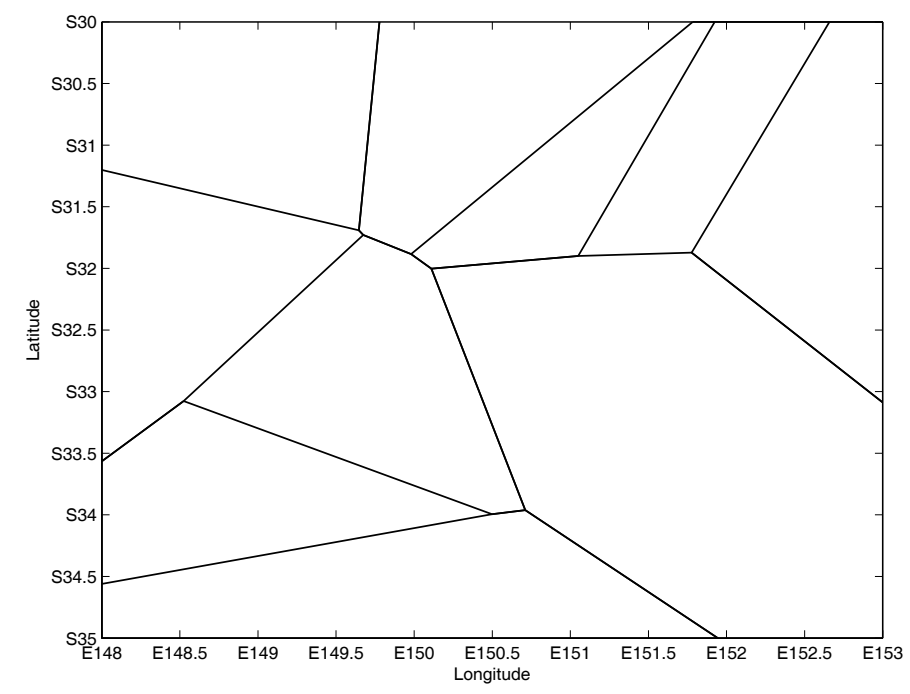

Fig. 6: A Balanced Airspace Sectorization Solution for Air Traffic 1

are: $F(W)=441.40$ traffic hits, $F(S F T)=488.49 \mathrm{sec}$, and $F(D)=0.07 \mathrm{~nm}$ which are all worse than the results achieved in Air Traffic 1. Therefore an airspace re-sectorization is required.

\section{B. Airspace Re-sectorization Results}

After airspace re-sectorization is undertaken in our second experiment part, the Pareto set is generated. The acceptable solutions (according to Equation 14) from the airspace resectorization are visualized in Figure 7, where the standard deviation of task load of each solution is less than 466.16 (calculated by $2330.8 \times 0.2$ ). The color of the points shows

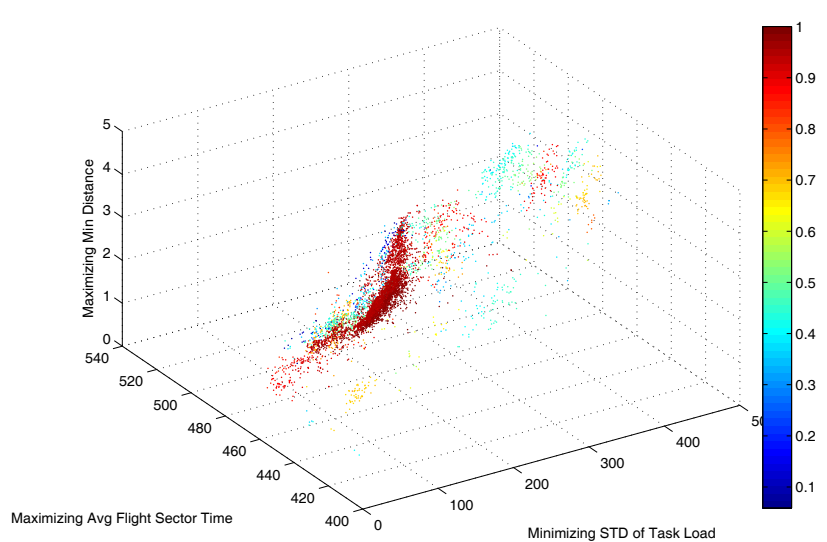

Fig. 7: The Task Load Balanced Solutions from the Pareto Set of Re-sectorization $(F(W)<466.16)$. The red dots mean higher similarity while the dark blue dots show mean lower similarity.

the similarity value of each solution: red dots mean higher similarity while the dark blue dots show lower similarity. As shown in the figure, the solutions with higher similarity are congested in the middle of the solution space. When the standard deviation of task load is reducing, the similarity is also decreasing. The similarity is increasing when the flight sector time and minimum distance between air traffic crossing points and sector boundaries are reducing. This shows that the airspace re-sectorization sacrifices the similarity to achieve the other three objectives. Maintaining similarity of sectors to the previous airspace sectorization means there is less flexibility to adjust the sector boundaries for other objectives. (The trade- 
offs between the other three objectives are analysed in [8].)

Five examples are selected to investigate our airspace resectorization model further:

- A solution that has the minimum standard deviation of task load.

- A solution that has the maximum average flight sector time.

- A solution that has the maximum minimum distance between air traffic crossing points and sector boundaries.

- A solution that has the maximum similarity to the previous sectorization.

- A solution that has the balanced achievements on all objectives.

\begin{tabular}{|l|r|r|r|r|}
\hline \multirow{2}{*}{ Solutions } & \multicolumn{4}{|c|}{ Objectives } \\
\cline { 2 - 5 } & Min. F(W) & Max. F(SFT) & Max. F(D) & Max. F(r) \\
\hline Best F(W) & 62.328 & 432.18 & 0.20 & 0.34184 \\
\hline Best F(SFT) & 350.21 & 536.82 & 0.04 & 0.37 \\
\hline Best F(D) & 456.13 & 469.36 & 4.49 & 0.38 \\
\hline Best F(r) & 348.34 & 508.46 & 0.09 & 0.85 \\
\hline Balanced & 187.89 & 502.58 & 1.82 & 0.68 \\
\hline Original & 441.41 & 488.49 & 0.07 & N/A \\
\hline
\end{tabular}

TABLE I: The Best and Balanced Solutions from Airspace Re-sectorization by 2D CVDM

Table I lists their fitnesses, and also shows the trade-off between the four objectives. The first three objective values from the original sectorization are also listed in the last line of the table. This table shows that all solutions from the resectorization are better than the original sectorization on at least one objective.

The sectors from these five solutions are plotted in Figure 8. The dashed lines are the original sector boundaries. Comparing the re-sectorization results with the original sectorization, the sector shapes from the first three solutions (with the minimum task load standard deviation, the maximum average flight sector time, and the maximum minimum distance between air traffic crossing points and sector boundaries respectively) are different from the original sectors, as illustrated in Figure 8a, $8 \mathrm{~b}$, and $8 \mathrm{c}$. The sector boundaries from the solution with the maximum similarity are very close to the original sectors, as shown in Figure 8d, which shows that the similarity measurement in our airspace re-sectorization model achieves good results. The similarity of the balanced solution is not bad where the sector shapes are similar to the original sectors, although some sectors located on the top are more different from the original ones.

\section{CONCLUSION}

In this paper, we propose an approach for Dynamic Airspace Re-sectorization according to the different air traffic situations. This approach is a 2D airspace re-sectorization methodology, based on our Constrained Voronoi Diagram Model, with the consideration of ATC task load balancing, maximizing average flight sector time, maximizing the minimum distance between air traffic crossing points and sector boundaries, and also maximizing the similarity of re-sectorization to the previous airspace sectorization. A similarity metric for 2D airspace resectorization is mathematically modeled and developed. Since the multi-objective airspace partition is an NP-hard problem, an evolutionary computation technology NSGA-II is applied in our approach.

The experimental results presented in this paper show that our proposed airspace re-sectorization model can undertake continuous airspace sectorization according to different air traffic situations, and can achieve all four objectives. In our analysis, we found that our similarity metric for airspace re-sectorizations can provide the correct information to the optimization method and can guide it to better solutions in the landscape. The trade-off between objectives is also found in the experiment results.

The proposed airspace re-sectorization model is experimentally proved in this paper. However, at present it is only a $2 \mathrm{D}$ approach. To use it in a 3D airspace, several problems have to be solved first. The most important issue is how to measure the similarity between two sectorizations in $3 \mathrm{D}$, which we will investigate in our future work.

\section{REFERENCES}

[1] C. Brinton and S. Pledgie, "Airspace partitioning using flight clustering and computational geometry," in Digital Avionics Systems Conference, 2008. DASC 2008. IEEE/AIAA 27th, oct. 2008, pp. 3.B.3-1 -3.B.3-10.

[2] J. S. B. Mitchell, G. Sabhnani, J. A. Krozel, R. L. Hoffman, and A. Yousefi, "Dynamic airspace configuration management based on computational geometry techniques," in AIAA Guidance, Navigation and Control Conference and Exhibit, no. AIAA 2008-7225, Honolulu, HI, Aug 2008.

[3] C. Verlhac and S. Manchon, "Optimization of opening schemes," in Proceedings of the fourth USA/Europe Air Traffic Management R\&D Seminar, 2001.

[4] D. Delahaye, M. Schoenauer, and J.-M. Alliot, "Airspace sectoring by evolutionary computation," in IEEE World Congress on Computational Intelligence 1998., may 1998, pp. 218 -223.

[5] M. Xue, "Airspace Sector Redesign Based on Voronoi Diagrams," in Proc. of AIAA Guidance, Navigation, and Control Conference and Exhibit, 2008, pp. 18-21.

[6] H. Songchen and M. Zhang, "The optimization method of the sector partition based on metamorphic voronoi polygon," Chinese Journal of Aeronautics, vol. 17, no. 1, pp. 7-12, 2004.

[7] R. Kicinger and A. Yousefi, "Heuristic method for 3D airspace partitioning: Genetic algorithm and agent-based approach," in 9th AIAA Aviation Technology, Integration, and Operations Conference (ATIO) and Aircraft Noise and Emissions Reduction Symposium (ANERS), September 2009.

[8] J. Tang, S. Alam, C. Lokan, and H. A. Abbass, "A multi-objective approach for dynamic airspace sectorization using agent based and geometric models," Transportation Research Part C: Emerging Technologies, vol. 21, no. 1, pp. 89 - 121, 2012. [Online]. Available: http://www.sciencedirect.com/science/article/pii/S0968090X11001185

[9] K. Weiler and P. Atherton, "Hidden surface removal using polygon area sorting," SIGGRAPH Comput. Graph., vol. 11, pp. 214-222, July 1977. [Online]. Available: http://doi.acm.org/10.1145/965141.563896

[10] K. Deb, K. Sindhya, and T. Okabe, "Self-adaptive simulated binary crossover for real-parameter optimization," in GECCO '07: Proceedings of the 9th annual conference on Genetic and evolutionary computation. New York, NY, USA: ACM, 2007, pp. 1187-1194.

[11] K. Deb and M. Goyal, "A combined genetic adaptive search (geneas) for engineering design," Computer Science and Informatics, vol. 26, pp. 30-45, 1996.

[12] S. Alam, H. Abbass, and M. Barlow, "ATOMS: Air traffic operations and management simulator," IEEE Transactions on Intelligent Transportation Systems, vol. 9, no. 2, pp. 209-225, June 2008. 


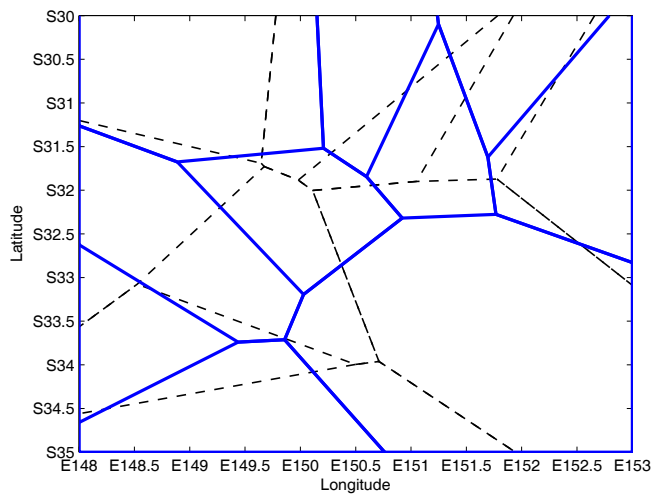

(a) Sectors with Min Task Load STD

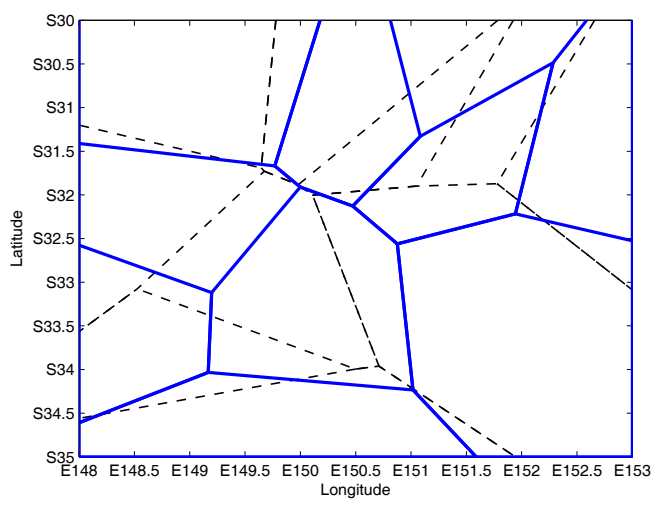

(c) Sectors with Max Distance

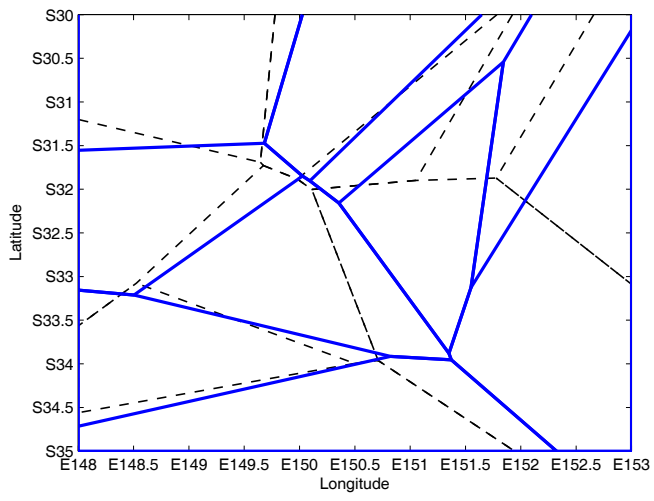

(b) Sectors with Max Flight Sector Time

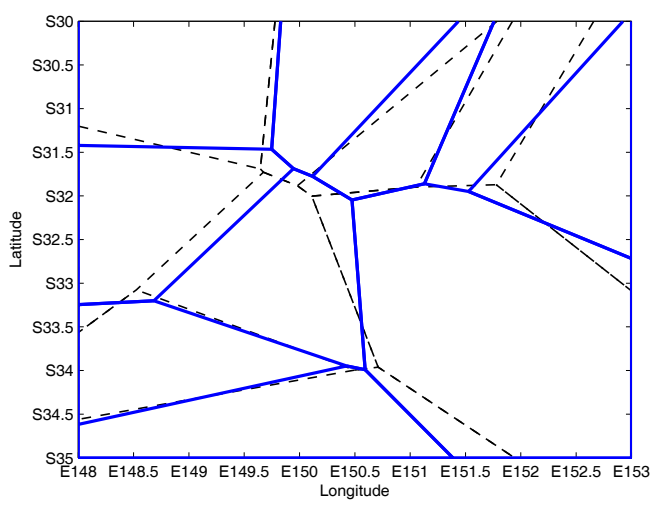

(d) Sectors with Max Similarity

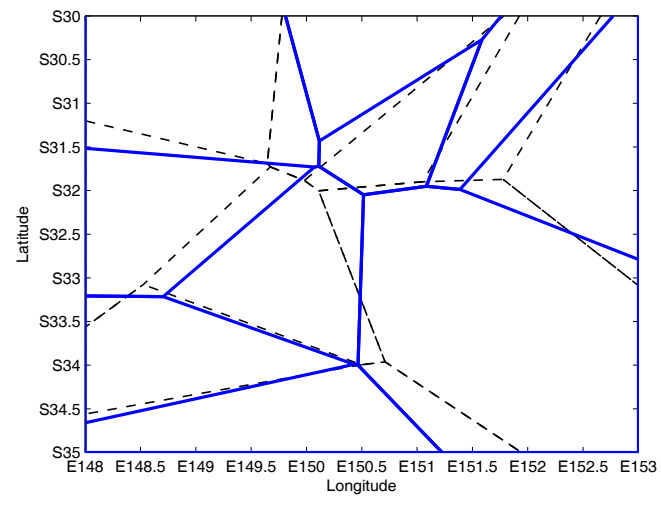

(e) Sectors with Balanced Objectives

Fig. 8: The Sectors of 5 Solutions from the Air Airspace Re-sectorization and Comparison with the Original Sectors 